\title{
Drug saves monkeys from close relative of Ebola
}

Animals spared from Marburg virus even when treated three days after infection.

\section{Erika Check Hayden}

20 August 2014

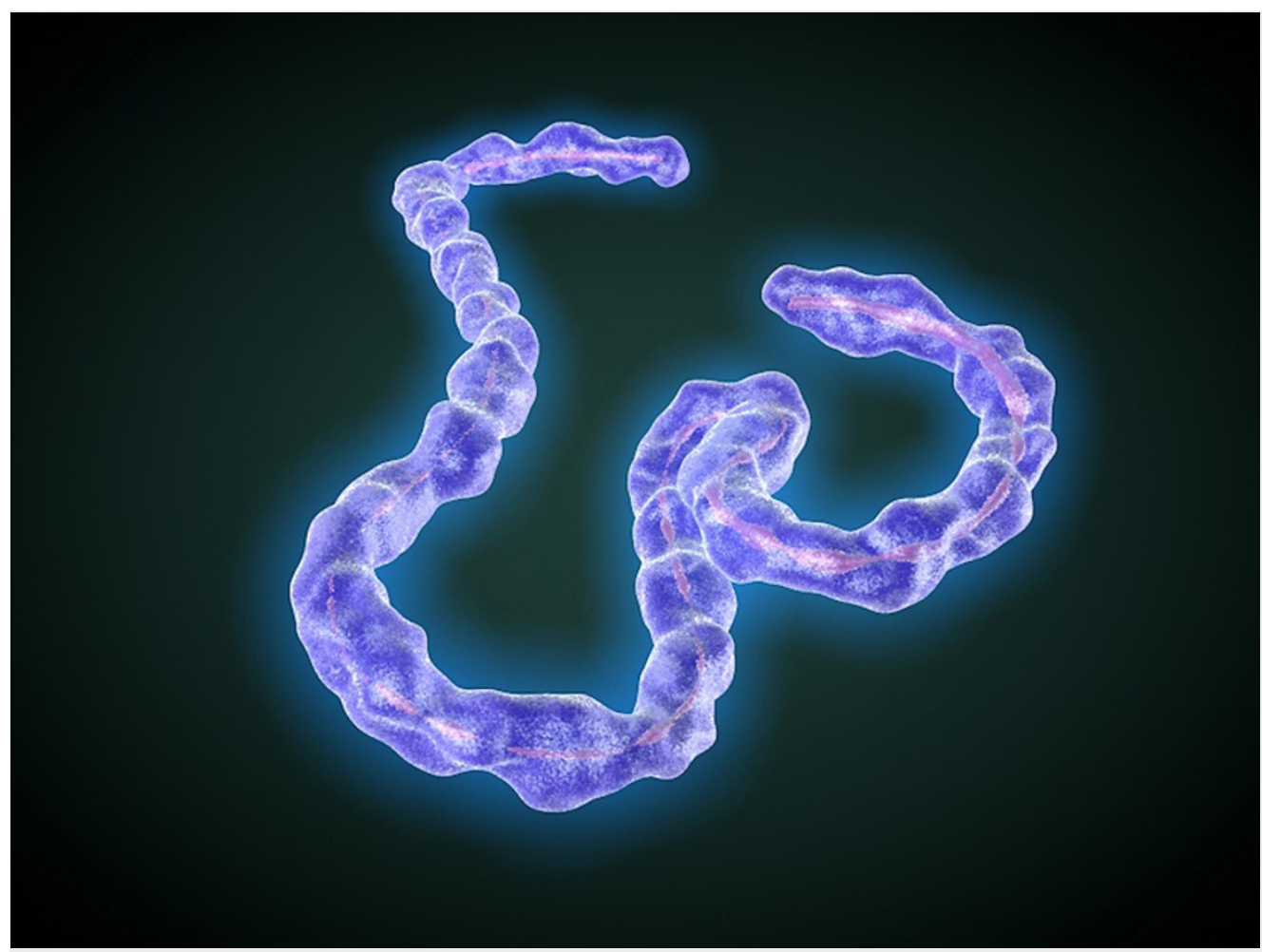

C. Bickel/Science Translational Medicine

The Marburg virus causes a lethal haemorrhagic fever.

A medicine administered days after infection can save monkeys from a deadly virus related to Ebola, researchers report in Science Translational Medicine ${ }^{1}$.

"This clearly starts to move into the realm of being a therapy, rather than a post-exposure treatment," says virologist Gene Olinger, principal science adviser for contract-research organization MRIGlobal in Kansas City, Missouri, who was not involved in the study. "It's a tougher point to intervene, so it's important that they've demonstrated this."

The drug, called TKM-Marburg, was developed by pharmaceutical company Tekmira in Burnaby, Canada, to treat Marburg virus. Like its close relative Ebola, the Marburg virus causes a lethal haemorrhagic fever; an outbreak in Angola in 2004-05 killed more than 90\% of people infected, and the virus is circulating in countries affected by the current Ebola outbreak, such as Sierra Leone ${ }^{2}$.

In the latest study, researchers used the Tekmira drug against Marburg in 16 rhesus macaques (Macaca mulatta), divided into four groups that each received the treatment at a different time: 30-45 minutes after infection, or one, two or three days after infection. All of the treated monkeys - including the four given the drug three days after exposure, roughly equivalent to day six of a human infection - survived, whereas four untreated animals died.

\section{Time sensitive}

Study author Thomas Geisbert, a microbiologist at the University of Texas Medical Branch in Galveston, says that this is the first time that a single drug has been shown to save animals from Marburg when it is administered days after the virus enters their bodies.

Currently the only way to confirm a Marburg or Ebola infection in humans is to wait several days until the virus has reached detectable levels in a person's blood.

The Tekmira drug uses pieces of genetic material — small interfering RNAs, or siRNAs — to disrupt the Marburg virus's ability to copy 
itself, thus stalling the course of infection. The siRNAs are encased in an envelope made of a fat-like material.

Some researchers say that the current study could aid in the decision about whether to use experimental medicines in the current Ebola outbreak. No medicines have yet been approved to treat the virus, but several are in development - including one, TKM-Ebola, made by Tekmira using similar techniques to TKM-Marburg. The World Health Organization (WHO) said on 12 August that the use of these unapproved medicines would be ethically acceptable in the current situation.

The TKM-Marburg research "shows that you have a window of opportunity when you can actually intervene before the tipping point when people are going to be too ill to recover", says Amesh Adalja, an infectiousdisease physician at the University of Pittsburgh Medical Center in Pennsylvania.

Experimental medicines are often available only in small quantities, so understanding exactly when the tipping point occurs could help researchers to decide which patients are good candidates for treatment, Adalja said. For instance, a Spanish priest infected with Ebola in Liberia was given one of the very existing few doses of an experimental monoclonal antibody cocktail — ZMapp, made by Mapp Biopharmaceutical of San Diego, California. He later died, possibly because he received the drug after he was too ill to benefit from it.

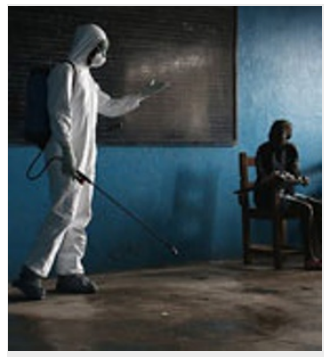

Nature special: Ebola outbreak in West Africa

"When you have scarce resources, you have to ask who is too sick to get better no matter what you give," says Adalja.

\section{Clinical trials}

Geisbert says that his group would like to test whether TKM-Ebola also works when given days after infection, but at the moment is limited in what it can do by lack of funding. TKM-Ebola has been shown to protect monkeys when a series of doses are given starting 30 minutes after the animals were infected ${ }^{3}$, and the drug was being tested in human safety trials that were stopped by US regulators in July because of safety concerns. But regulators said on 7 August that it could be used in people with Ebola.

Julie Rezler, Tekmira's director of investor relations and corporate communications, says that the company is "speaking with several governments and NGOs [non-governmental organizations] in various countries, as well as WHO" about TKM-Ebola. But the company has not said whether any of the groups have asked for the medicine.

In addition to the Spanish priest, two American health workers and three Liberian health workers have received doses of ZMapp. In March, Geisbert and other researchers received a US\$26-million grant from the US National Institute of Allergy and Infectious Diseases to study a combination of TKM-Ebola and ZMapp.

One hurdle in the development of drugs for viral haemorrhagic fevers has been the need to create separate drugs for the many strains of each virus that exist. But in their paper, Geisbert and his team describe how TKM-Marburg protects against several different strains. Geisbert says that if he were to get appropriate funding, clinical trials of the Marburg drug could be conducted within a year.

Nature | doi:10.1038/nature.2014.15745

\section{References}

1. Thi, E. P. et al. Sci. Transl. Med. 6, 250ra116 (2014).

2. Schoepp, R. J., Rossi, C. A., Khan, S. H., Goba, A. \& Fair, J. N. Emerg. Infect. Dis. 20, 1176-1182 (2014).

3. Geisbert, T. W., et. al. Lancet 375, 1896-1905 (2010). 